\title{
Neu auftretende Thoraxdeformierungen bei Kindern und Jugendlichen
}

\author{
H. Schnyder-Etienne
}

Bei den schulärztlichen Untersuchungen entdeckten wir bei unseren Kindern und Jugendlichen in den letzten 3-5 Jahren nebst der bekannten Zunahme von Rückenschmerzen und Rückenschäden auch eine Zunahme von neu aufgetretenen Thoraxdeformierungen.

Korrespondenz:

Dr. med. Hanna Schnyder-Etienne Praxis Chabloz

CH-3953 Leuk-Stadt

Tel. 0274731306

Fax 0274734281

E-Mail:

h.schnyder.etienne@bluewin.ch
Seit 12 Jahren bin ich Schulärztin in der Region Leuk (Einzugsgebiet von etwa 800 Kindern und Jugendlichen). Mit grosser Besorgnis beobachteten wir in den letzten Jahren die zunehmenden Haltungs- und Rückenschäden bis hin zum «Haltungszerfall» unserer Kinder und Jugendlichen. Dies ist ein Phänomen, welches nicht nur bei uns, sondern gesamtschweizerisch beobachtet wird.

Trotz verschiedener Kampagnen (z. B. «Sitzen als Belastung» vom Schweizerischen Verband für Sport in der Schule SVSS) scheint die Thematik des gesunden Rückens und der gesunden Haltung bei den Eltern, Lehrpersonen und zum Teil auch bei uns Ärztinnen und Ärzten verlorengegangen zu sein - dies ganz im Gegensatz zur Prophylaxe bei den Zähnen, wo man kaum mehr

\section{Abbildung 1}

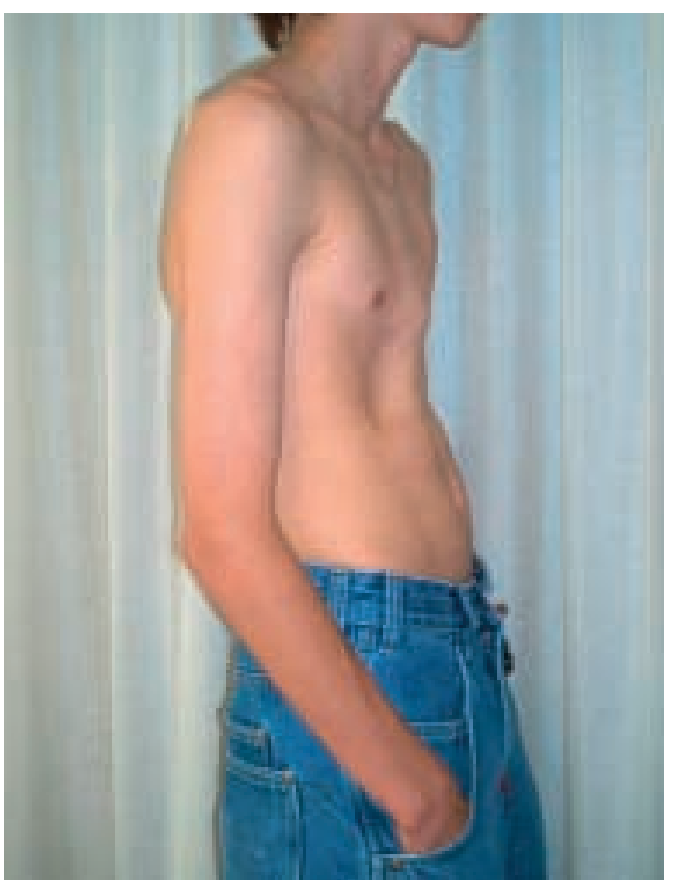

Jugendliche ohne Zahnkorrekturen sieht. Wie haben es die Zahnärzte geschafft, den Eltern absolut klarzumachen, dass sie ihre Kinder meist von sich aus zur Kontrolle der Zahnstellung anmelden, die Kinder und Jugendlichen dies auch wünschen und das ganze noch grossteils selbst finanziert werden muss? Wir verhalten uns, als ob man im Notfall den Rücken und nicht die Zähne ersetzen könnte!

Erstmals in der menschlichen Entwicklung kompensieren unsere Kinder und Jugendlichen das lange Sitzen in der Schule nicht mit körperlicher Aktivität zu Hause, sondern sie sitzen weiter (Fernsehen, Computer, Internet, Gameboy usw.) und dies häufig über Stunden in einer miserablen Stellung.

Vor etwa 5 Jahren beobachtete ich in der herbstlichen Schuluntersuchung der 2. Klassen der Orientierungsschule (13- bis 15jährige) einen Jugendlichen mit neu aufgetretener, unschöner und absolut asymmetrischer Thoraxdeformierung (im Ansatz an eine Trichterbrust erinnernd). Wie konnte dies passieren, hatte ich ihn doch

Jugendlicher mit neu aufgetretener Thoraxdeformierung, stehend und sitzend.

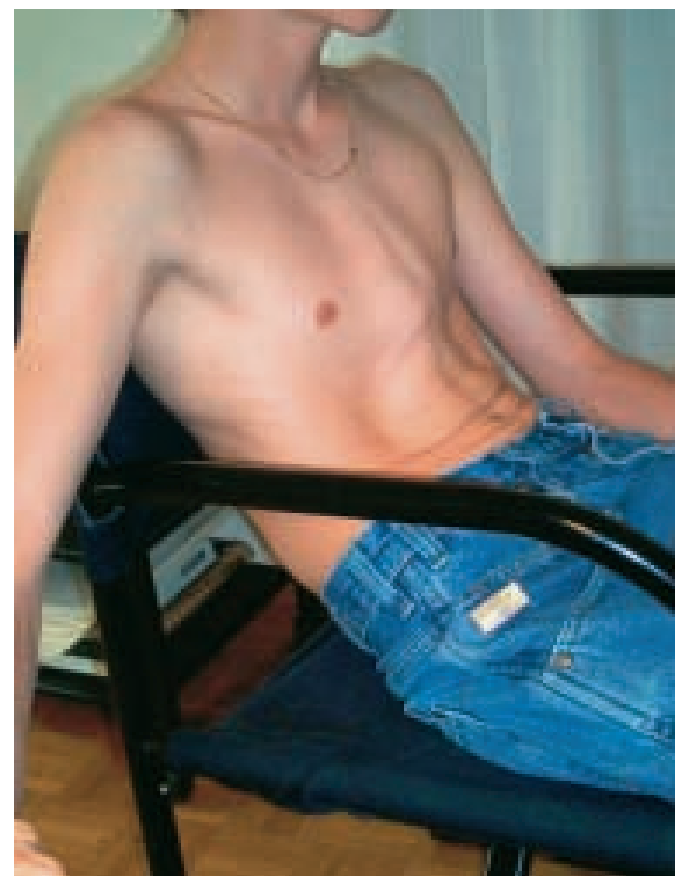


vor den Sommerferien in meiner Praxis genau untersucht und keinerlei Deformierungen festgestellt?

Um mir einen Überblick zu verschaffen, untersuchte und dokumentierte ich alle 2. Klassen der Orientierungsschule der Region Leuk. Von 78 Jugendlichen klagten 58\% über Rückenschmerzen, 6 Jugendliche $(7,7 \%)$ über chronische Schmerzen (täglich, länger als 3 Monate dauernd). Keiner dieser Jugendlichen hatte wegen dieser Beschwerden einen Arzt konsultiert, als wäre es normal, in diesem Alter Rückenschmerzen zu haben.

Vier Jugendliche zeigten Brustverformungen. Bei zweien von diesen Jugendlichen waren die Verformungen neu aufgetreten, bei den andern beiden hatten sich die bereits vorher bestandenen Thoraxnormvarianten in der Adoleszenz massiv verschlimmert bis hin zu Deformierungen. Gemeinsam war diesen Jugendlichen ein schlanker Körperbau und ein relativ starker Wachstumsschub. Zwei von ihnen trieben Sport (Fussball), zwei nicht.

Die wichtigste Gemeinsamkeit unter diesen Schülern bestand in der «coolen» schlaffen Haltung, sowohl im Stehen wie auch in ihrer gekrümmten Sitzhaltung, die sie wohl täglich über Stunden einnehmen. Auch eine Stunde Sport pro Tag (im optimalen Fall) konnte das «Negativkorsett» dieser schlechten Sitzhaltung über Stunden nicht korrigieren.

Heute sehen wir Kinder, die diese Verformungen im Ansatz bereits in der 4. bis 6. Primarklasse aufweisen. Aus ihren Verformungen kann man auf ihre Sitzhaltung schliessen.

Anscheinend bewirkt (nebst anderen Faktoren wie z.B. fehlende Belastung des Oberkörpers in unserer Gesellschaft mit unterentwickelten Muskeln) das monotone Verharren ohne Unterbrechung in einer derart schlechten Haltung eine enorme statische Belastung nicht nur auf die wachsende Wirbelsäule, sondern auch auf den Thorax, so dass es zu solchen Deformierungen kommt. Die Brustdeformierung kann sich über Monate ankündigen oder in kurzer Zeit auftreten, so als würde der Thorax dem Druck plötzlich nicht mehr standhalten und nachgeben. Die Zahnärzte benutzen dieses Druckprinzip sehr erfolgreich bei ihrer Arbeit mit Zahnspangen!

In meinem Kollektiv entwickelten etwa 1-2\% der Schulkinder eine Thoraxdeformierung. Die meisten Jugendlichen hatten wegen dieser Beschwerden keinen Arzt konsultiert. Wir finden sie in Reihenuntersuchungen der Schulen.

Es scheint uns, dass die Rückenschäden und die Thoraxverformungen sich vor allem in der Pubertät mit rasantem Tempo verschlechtern. In der diesjährigen Schuluntersuchung zeigte ein 15jähriges Mädchen einen Skoliosewinkel von 40 Grad bei bis dahin unauffälligem Rücken (letzte Konsultation vor 1 1/2 Jahren). Häufig werden genau in diesem Alter die Jugendlichen nicht mehr jährlich untersucht!

Unsere Kinder bewegen sich nicht mehr genügend, um ungeschädigt durch unser Schulsystem zu kommen. Die Schulzeiten sind zu lang und der Fernseher und Computer sind bereits in die Kinderzimmer unserer kleinsten Kinder vorgedrungen. Unsere Jugendlichen surfen und chatten zum Teil über Stunden im Internet. Zuallerletzt haben unsere Kinder keinen wirklichen Schulweg mehr. In unserem Kollektiv ist der Schulweg der Primarschüler im Durchschnitt 9 Minuten (!), der unserer Orientierungsschüler 7 Minuten (bis zum Schulbus). 50\% unserer Primarschulkinder der 2. Klassen werden von den Eltern in die Schule chauffiert, bei den 4 . Klassen sind es gar $60 \%$. Mit unserem Verhalten und dem Schulbussystem berauben wir unsere Kinder und Jugendlichen des Gratistrainings eines Schulweges, der gelaufen wird mit all seinen positiven Auswirkungen auf die Gesundheit.

Wir haben an unseren Schulen im Jahr 2003/2004 ein Gesundheitsprojekt «Fitte Kids biwegt geits bessär» lanciert. Das Projektziel ist, mehr Bewegung, Haltung, Körperbewusstsein und Entspannung in den Schulalltag zu bringen. Unser Projekt soll nachhaltig bleiben und stufengerecht konzipiert sein (vom Kindergarten bis ins 9. Schuljahr).

Nach gezielter Weiterbildung von rund 80 Lehrpersonen der Gemeinde Leuk und der Regionsgemeinden, der direkten Umsetzung in den Unterricht (Wissensvermittlung, bewegter Unterricht, Reaktivierung der Pausen, Schaffung von Bewegungsräumen in der Schule, auf den Strassen, «Laufen statt Fahren») und einer Information an die Eltern haben wir unser erstes Projektjahr ausgewertet. Wir fragten unsere Kinder und Jugendlichen unter anderem, wie es ihnen nach einem Jahr Projektarbeit gehe. In der 1 . OS (Orientierungsschule) gaben $24 \%$, in der 2 . OS 26\% eine Verbesserung ihrer Rückenschmerzen an. Dies entspricht etwa unseren Erwartungen für dieses schwierige Alter. Die Verbesserungen in der 4. PS (Primarschule) mit 71\%, in der 6. PS gar mit $85 \%$ und in der 3 . OS mit $56 \%$ waren unerwartet hoch ausgefallen.

Unsere Arbeitshypothese, dass die gezielte Weiterbildung der Lehrpersonen und die Information an die Eltern die gesundheitliche Situation unserer Kinder verbessern, hat sich bestätigt. Nach einem Projektjahr geht es vielen Jugendlichen in bezug auf ihre Rückenschmer- 
zen besser oder ganz gut, was nicht nur für den einzelnen sehr wichtig ist, sondern auch der Gesellschaft (später resultierende) Gesundheitskosten in Millionenhöhe erspart.

Rückenprobleme gehören bei der IV zu den häufigsten Diagnosen! Rückenprobleme und Depressionen treten relativ häufig gemeinsam auf.

Wir Ärztinnen und Ärzte sollten aktiver in die Diskussion der Gesundheitsförderung in den Schulen und zu Hause eingreifen.

An der Generalversammlung der Oberwalliser Ärztegesellschaft Anfang April 2005 haben wir ein Bewegungsmanifest verabschiedet, das unter anderem folgendes fordert:

1. Die Aufklärung der Eltern als erste Bewegungserzieher ihrer Kinder.

2. Die Ausbildung und Weiterbildung aller Lehrpersonen inklusive der Studierenden der pädagogischen Hochschule Wallis in wichtigen Gesundheitsfragen wie Bewegungsmangel, Rücken- und Thoraxprobleme, Übergewicht, Ernährung usw.

3. Die Verantwortlichen für Erziehung und Gesundheit im Kanton Wallis und in den Gemeinden fördern gemeinsam mit den Lehrpersonen Bewegungsprogramme in den Schulen.

4. Bewegung und Sport muss den Hauptfächern gleichgestellt werden.

5. Eine Stunde Bewegung pro Tag ist als Minimum zu fordern.

6. Die Umsetzung des bewegten Unterrichtes muss in allen Schulen gefördert werden.

Wenn wir die Zeichen der Zeit nicht erkennen, explodieren unsere Gesundheitskosten, und unsere Kinder und Jugendlichen sind kränker als die Generation ihrer Eltern. Eine gemeinsame Offensive ist dringend nötig, nicht nur bei den Schulärztinnen und Schulärzten.

Wir freuen uns über Anregungen. Falls Sie sich über unser Projekt ein Bild machen wollen, wenden Sie sich bitte an die Schuldirektion Leuk (schuldirektion.leuk@gmx.ch).

\section{Literatur}

- Zahner L, Phüse U, Stüssi C, Schmid J, Dösegger A. Aktive Kindheit - gesund durchs Leben. Handbuch für Fachpersonen. Bern: Bundesamt für Sport, Institut für Sport und Sportwissenschaften, Stiftung für Schadensbekämpfung der Winterthur Versicherungen; 2004.

- SVSS. Sitzen als Belastung - wir sitzen zu viel. Aspekte des Sitzens; eine Lehrunterlage. Olten: SVSS; 1991.

- Fakten zur gesundheitlichen Bedeutung von Bewegung und Sport im Jugendalter; eine gemeinsame Stellungnahme aus wissenschaftlicher Sicht vom BASPO, BAG, SGPG, SGP, SGSM, Netzwerk Gesundheit und Bewegung Schweiz; Schweiz Z Sportmed Sporttraumatol 1999;47:921-3.

- Hancox RJ et al. Association between child and adolescent television viewing and adult health: a longitudinal birth cohort study. Lancet 2004; 364:257-62.

- Breithecker D. Lust auf Schule - Lust auf Lernen. Mehr Gesundheit und Wohlbefinden am «Arbeitsplatz Schule» - ein Projektbericht. Haltung und Bewegung 2000;4(20):27-33.

- Küster M, et al. Zu Hause nur TV und Computer!? Gesundheitsfolgen des Freizeitverhaltens heutiger Jugendlichen. Pädiatrie 2003;(4).

- Watson KD, Papageorgiou AC, Jones GT, Taylor S, Symmons DP, Silman AJ, Macfarlane GJ. Low back pain in schoolchildren; the role of mechanical and psychosocial factors. Arch Dis Child 2003; $88 ; 12-7$.

- $\quad$ Etienne Klemm R. Die Kraft der inneren Bilder; Entstehung, Ausdruck und therapeutisches Potential. Basel: Schwabe Verlag; 2003.

- Bewegung und Gesundheit. Lehrmittel. Olten: SVSS; 2002.

- Bucher W (Hrsg.). Buchreihe «Spiel- und Übungsformen». Schorndorf: Verlag Hofmann; 2000.

- Gesundheitsförderung in der Schule - «Balance»; Lehrmittelverlag des Kantons Aargau.

- $\quad$ Illi U, Breithecker D, Mundlinger S (Hrsg.). Bewegte Schule - Gesunde Schule. Zürich/ Wiesbaden/Graz; 1998. 